\title{
MARCH9 Suppresses Lung Adenocarcinoma Progression by Downregulating ICAM-1
}

\author{
Qi-ming Shen ${ }^{a, b}$ Hao-you Wang ${ }^{a, b}$ Shun $X u^{a, b}$ \\ aChina Medical University, Shenyang, ${ }^{b}$ Department of Thoracic Surgery, the First Hospital of China \\ Medical University, Shenyang, China
}

\section{Key Words}

Lung adenocarcinoma $\cdot \mathrm{MARCH} \cdot$ Invasion $\bullet$ Prognosis

\begin{abstract}
Background/Aims: To investigate the clinical significance and functional mechanisms of membrane-associated RING-CH protein 9 (MARCH9) in lung adenocarcinoma (LAC). Methods: Immunohistochemistry staining was performed to explore the expression of MARCH9 in LAC tissues and adjacent normal lung tissues. Patients' prognosis was evaluated using overall survival. The prognostic role of MARCH9 was tested with univariate and multivariate analyses. To confirm the effect of MARCH9 in cell proliferation and invasion, overexpression of MARCH9 was induced in two LAC cell lines. Cell cycle, apoptosis, migration, invasion, and immunoprecipitation experiments were performed to further explore the signaling pathways involved. Results: Analysis of a series of 143 clinical samples revealed that MARCH9 was downregulated in tumor tissues compared with normal lung tissues, and this was closely associated with lymph node metastasis $(P=0.004)$. Univariate and multivariate analyses indicated that MARCH9 was an independent prognostic biomarker for LAC; low MARCH9 expression indicated poor overall survival. Cellular studies with A549 and H1299 cells demonstrated that $\mathrm{MARCH} 9$ can attenuate tumor migration and invasion but had little effect on cell cycle or apoptosis. Moreover, an interaction between MARCH9 and ICAM-1 protein was identified, and overexpression of MARCH9 was found to attenuate the oncogenic effect of ICAM-1, suggesting that MARCH9 may inhibit tumor progression by downregulating ICAM-1 signaling. Conclusion: MARCH9 downregulation in LAC tissues correlated with poor clinical outcomes. MARCH9 may serve as a novel biomarker and potential therapeutic target for LAC.
\end{abstract}




\section{Cellular Physiology Cell Physiol Biochem 2018;50:92-107

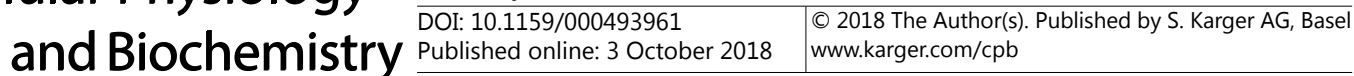 Shen et al.: MARCH9 Inhibits LAC Progression}

\section{Introduction}

Lung cancer is one of the most common tumor types worldwide and is the leading cause of cancer-related mortality [1]. The histological classification of lung cancer has two major subtypes, non-small cell lung cancer (NSCLC) and small cell lung cancer. NSCLC is typically carries a poor prognosis, with 5-year overall survival at around 15\% [2]. Usually originating from peripheral lung tissues, lung adenocarcinoma (LAC) comprises more than $40 \%$ of lung cancers and has a high prevalence of distant metastasis [3]. The established curative method for managing LAC is surgical treatment. Unfortunately, it is still difficult to predict clinical outcomes even for patients who undergo tumor resection [4]. Also, despite the considerable advances that have been made in early detection and targeted therapies for lung cancer, patients with advanced disease still end up with unfavorable outcomes. The predominant factors for poor prognosis include metastasis, early recurrence, and poor response to adjunctive therapy. Therefore, better understanding of the metastasis mechanisms and identification of novel biomarkers are essential to help predict the prognostic patterns of LAC patients as well as develop novel therapeutic interventions.

Major histocompatibility complex (MHC) proteins, including MHC class I and MHC class II, play important roles in initiating immune response and are highly expressed in antigen-presenting cells [5]. The membrane-associated RING-CH (MARCH) proteins were first identified as ubiquitination regulators of MHC proteins, modulating the overall levels of MHC proteins on the cell surface [6, 7]. The MARCH family comprises 11 members, MARCH1 to MARCH11. Besides the roles in the immune system, MARCH proteins are also involved in other cellular functions [8]. For example, MARCH1 was reported to regulate insulin sensitivity by controlling the level of cell surface insulin receptors [9]. MARCH2 can promote endocytosis and degradation of beta2-adrenergic receptors and is thought to be involved in regulating cardiovascular function [10]. MARCH5 is located in the mitochondria and controls mitochondrial fission and cell sensitivity to stress-induced apoptosis [11]. MARCH7 regulates $\mathrm{T}$ cell proliferation and neuronal development and participates in membrane trafficking and protein degradation.

Accumulating evidence suggests that the MARCH proteins can also regulate tumor development and progression. For example, MARCH1 knock out suppresses proliferation, migration, and invasion of ovarian cancer cells by suppressing the NF- $\mathrm{\kappa B}$ and Wnt/ $\beta$-catenin pathways [12]. Similarly, MARCH2 silencing inhibits the growth of colon cancer cells via induction of endoplasmic reticulum stress [13]. MARCH5 and MARCH7 can both promote the processes of migration and invasion in ovarian cancer cells $[14,15]$. High expression of MARCH8 was also observed in esophageal tumors and was associated with tumor aggression [16]. However, at present, there is no evidence about the role of MARCH9 in malignancy.

In the present study, we first determined the mRNA and protein levels of MARCH9 in LAC tissues and adjacent lung tissues. Statistical analysis confirmed its predictive role as an independent prognostic factor for poor overall survival in LAC patients. We also performed cellular experiments to explore its functional mechanisms. MARCH9 significantly inhibited the processes of migration and invasion in LAC cells but had little effect on cell proliferation or apoptosis. Of note, we further found that MARCH9 could downregulate the protein levels of Intercellular adhesion molecule 1 (ICAM-1), and this maybe the underlying mechanism in repressing tumor metastasis. Our results not only provide initial evidence about the suppressive role of MARCH9 in tumor progression, but also reveal a novel MARCH9-ICAM-1 signaling pathway in tumor cells, and may therefore help direct targeted therapeutic strategy for LAC patients. 


\section{Cellular Physiology Cell Physiol Biochem 2018;50:92-107 and Biochemistry Published online 3 October $2018 \begin{aligned} & \text { DOI: 10.1159/000493961 } 2018 \text { The Author(s). Published by S. Karger AG, Basel } \\ & \text { www.karger.com/cpb }\end{aligned}$ Shen et al.: MARCH9 Inhibits LAC Progression}

\section{Materials and Methods}

Patients and samples

A total of 143 patients (43 women, 100 men) diagnosed with primary LAC were recruited from our hospital (Shenyang, China) between March 2005 and March 2014. All patients underwent surgical resection at our hospital and all declined preoperative drug treatment. Retrospectively collected clinical information included age, smoking history, tumor size, histological differentiation grade, lymph node metastasis, and TNM staging. Complete follow-up details were obtained for all LAC patients and were included in overall survival analysis. We collected formalin-fixed paraffin-embedded (FFPE) tissues as well as 12 pairs of freshly resected LAC and adjacent normal lung tissue samples, which were flash-frozen and stored in liquid nitrogen for further use.

\section{Immunohistochemistry (IHC)}

IHC experiments and staining evaluation were performed as described elsewhere [17]. Briefly, FFPE specimens were cut into $4 \mu \mathrm{m}$ serial sections, deparaffinized, blocked, and incubated at $4^{\circ} \mathrm{C}$ overnight with the primary antibodies (MARCH9, ab99231, Abcam, Cambridge, UK; ICAM-1, ab2213, Abcam), followed by incubation with horseradish peroxidase-labeled secondary. Two independent pathologists analyzed immunoreactivity in at least six randomized visual fields based on both staining intensity and percentage of positively stained cells. Briefly, the percentage of positively stained cells was scored as 1 (0-25\%), 2 (25$50 \%), 3(50-75 \%)$, or 4 (75-100\%). The staining intensity score was classified as 1 (unstained, negative), 2 (slightly stained, light yellow), 3 (medium stained, dark yellow), or 4 (strongly stained, dark brown). The final IHC score was calculated by multiplying these two scores (range 1-16). Based on the IHC score, we then divided the patients into a high MARCH9 expression group (IHC score $\geq 9, \mathrm{n}=68$ ) and a low MARCH9 expression group (IHC score $<9, \mathrm{n}=75$ ).

\section{Quantitative real-time polymerase chain reaction ( $R T-q P C R$ )}

Total RNA was extracted from fresh-frozen tissues using TRIZOL reagent (Invitrogen, Carlsbad, CA). Reverse transcription PCR was performed using PrimeScript RT reagent Kit (TaKaRa, Dalian, China) according to the manufacturer's instructions. Real-time PCR was carried out to quantify MARCH9 transcript level using SYBR Premix Ex Taq II (RR420A; TaKaRa) according to a standard protocol [18]. The housekeeping gene GAPDH was used as normalization control. The following primers were used: MARCH9 forward: $5^{\prime}-$ AATCCGCTGGATCAGTGAGAGG-3'; MARCH9 reverse: 5'-GCAGCAATCTGGACCTTCTCGA-3'; GAPDH forward: 5'-CCTGCACCACCAACTGCTTA-3'; and GAPDH reverse: 5'-GGCCATCCACAGTCTTCTGAG-3'.

\section{Western blot}

Resected tissues or cultured cells were lysed with lysis buffer. The supernatant of the lysate was collected by centrifuging at 13,000 rpm for $30 \mathrm{~min}$. After protein quantification, about $30 \mu \mathrm{g}$ of total protein was loaded onto $10 \%$ sodium dodecyl sulfate polyacrylamide gel electrophoresis and transferred to polyvinylidene difluoride membranes. The membranes were blocked with $5 \%$ bovine serum albumin for $1 \mathrm{~h}$ at room temperature and then incubated with specific antibodies (MARCH9, $\beta$-actin, ICAM-1, GTP-RhoA, RhoA, and Flag; all purchased from Abcam) at $4{ }^{\circ} \mathrm{C}$ overnight. Membranes were subsequently incubated with secondary antibodies for another $1 \mathrm{~h}$ at room temperature. Protein bands were visualized using an ECL western blotting kit (Thermo Fisher Scientific, Pittsburgh, PA, USA).

\section{Cell culture}

The human normal bronchial epithelial cell line (HBE) and two LAC cell lines (H1299 and A549) were purchased from the Institute of Biochemistry and Cell Biology of the Chinese Academy of Sciences (Shanghai, China). All the cells were cultured in RPMI 1640 medium supplemented with 10\% fetal bovine serum (FBS; GIBCO, Gaithersburg, MD, USA) and 1\% penicillin-streptomycin. All cells were maintained at $37^{\circ} \mathrm{C}$ in a humidified atmosphere with $5 \% \mathrm{CO}_{2}$. 


\section{Cellular Physiology Cell Physiol Biochem 2018;50:92-107

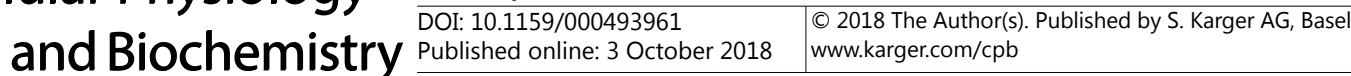 \\ Shen et al.: MARCH9 Inhibits LAC Progression}

\section{Plasmids and transfection}

siRNA targeting human MARCH9 was purchased from Sigma (Cat.: sc-95940). Flag-tagged MARCH9 and ICAM-1 constructs were inserted into pcDNA3.0 vector, respectively. The plasmids were both constructed by GenePharma (Shanghai, China) and verified with DNA sequencing by BGI (Beijing, China). Transfection was performed using Lipofectamine ${ }^{\circledR} 2000$ (Invitrogen; Thermo Fisher Scientific) according to the manufacturer's instructions. After $24 \mathrm{~h}$, transfected cells were partially harvested to assess the transfection efficiency; the remaining cells were expanded and subjected to functional assays.

\section{MTT assay}

MTT assays were performed to assess cell proliferation [19]. Briefly, 3000 cells in $200 \mu \mathrm{L}$ FBScontaining DMEM were seeded into each well of 96-well plates and cultured for several days. Triplicate wells from each transfection group were assessed every day (days 0-4). At the designated time points, 100 $\mu \mathrm{L}$ fresh medium containing MTT $(5 \mathrm{mg} / \mathrm{mL})$ was added to each well and the cells were incubated at $37^{\circ} \mathrm{C}$ for $4 \mathrm{~h}$. The medium was then replaced with $150 \mu \mathrm{L}$ DMSO and the cells were shaken at room temperature for $10 \mathrm{~min}$ to dissolve crystals. Absorbance was measured at $490 \mathrm{~nm}$. Each experiment was performed in triplicate, and results are presented as the mean \pm SD.

\section{Colony formation}

Colony formation assay was performed on a 6-well plate for 2 weeks. The colonies were fixed with paraformaldehyde and then stained with $0.1 \%$ crystal violet (Sigma-Aldrich, St Louis, MO, USA). Visible colonies were counted manually. All experiments were performed at least three times.

\section{Polyubiquitination analysis}

To test whether MARCH9 regulates the K48-linked poly-ubiquitination of ICAM-1, we transfected cells with calmodulin binding peptide (CBP)-tagged ICAM-1, HA-tagged ubiquitin (K48), as well as Flag-tagged MARCH9 plasmids. After culturing for $48 \mathrm{~h}$, cells were harvested and subjected to immunoprecipitation using CBP affinity beads. The pull-down products were then tested by both anti-HA and anti-CBP antibodies as described above.

Cell cycle

Cell cycle phases (G1, S, or G2 phase) were identified based on different DNA contents using the fluorescent dye propidium iodide (PI; Sigma Aldrich) according to the standard procedure. Briefly, cells were fixed with $70 \%$ cold ethanol $(700 \mu \mathrm{L})$ at $4^{\circ} \mathrm{C}$ for $4 \mathrm{~h}$ and then washed and stained with PI at $37^{\circ} \mathrm{C}$ in the dark for $30 \mathrm{~min}$. After filtering through a nylon mesh ( 400 screen meshes), cells were introduced to a fluorescence-activated cell sorting (FACS) flow cytometer (BD Biosciences, Franklin Lakes, NJ, USA). The ratio of cells in various cell cycle phases were counted and analyzed. All experiments were performed in triplicate and repeated at least three times.

\section{Apoptosis}

The Annexin V-FITC Apoptosis Detection kit (Calbiochem, Darmstadt, Germany) was used to assess apoptosis in transfected cells. Briefly, cultured cells were suspended, centrifuged and washed with phosphate buffered saline and then resuspended in cold Binding Buffer (Santa Cruz Biotechnology, Dallas, TX, USA) supplemented with $1.25 \mu \mathrm{L}$ Annexin V-FITC reagent according to the manufacturer's instructions. Flow cytometric analysis was also performed using a BD FACS flow cytometer. All data were quadrantally analyzed to distinguish early apoptotic cells, late apoptotic cells, dead cells, and viable cells. The apoptotic index was calculated by combining the percentage of early and late apoptotic cells. All experiments were performed in triplicate and repeated at least three times.

\section{Wound healing}

Transfected cells were cultured in 6 -well plates at $2.5 \times 10^{5}$ cells per well. When the cells reached $80-90 \%$ confluency, the cells were scratched using sterile $200 \mu \mathrm{L}$ pipette tips. The cell culture medium was refreshed to remove floating cells, and the width of the scratch was immediately observed with an inverted microscope as the original control. At the 24 -h and 48-h time points, the width of the scratches was 


\section{Cellular Physiology Cell Physiol Biochem 2018;50:92-107 \begin{tabular}{l|l|l} 
and Biochemistry Published online: 3 October 2018 & $\begin{array}{l}\text { @ } 2018 \text { The Author(s). Published by S. Karger AG, Basel } \\
\text { www.karger.com/cpb }\end{array}$ \\
\hline
\end{tabular} \\ Shen et al.: MARCH9 Inhibits LAC Progression}

observed; percent wound closure was calculated by comparing with the original width. Each experiment was repeated in triplicate.

\section{Transwell migration and Matrigel invasion assays}

Migration assays were performed using transwell chambers with $8.0 \mu \mathrm{m}$ pore membrane inserts [20]. Transfected Hep3B and Huh7 cells were seeded in a separate culture well and allowed to undergo migration in a $37^{\circ} \mathrm{C}$ incubator for $24 \mathrm{~h}$. The cells were cultured with $10 \%$ FBS in the upper chamber and DMEM containing 20\% FBS in the lower chamber. After $24 \mathrm{~h}$ of culture, cells on the lower surface of the membrane were fixed and stained with crystal violet. For each well, 10 random fields were counted and the average number of cells was determined. Each experiment was performed in triplicate and repeated for at least three times. For the invasion assays, transwell chambers were precoated with Matrigel $(0.4 \mathrm{mg} / \mathrm{mL})$ before seeding cells; the invasion period was for $48 \mathrm{~h} \mathrm{[21].}$

\section{Statistical analysis}

Statistical analysis was performed using SPSS version 18.0 (IBM, New York, NY). Pearson chi-square tests were used to compare differences between the high-MARCH9 expression group and low-MARCH9 expression group. Survival curves were plotted with the Kaplan-Meier method and compared using the logrank test. Significant factors identified by the univariate models were further entered into a multivariable Cox regression model to test their independent roles. For all tests, $P<0.05$ was considered statistically significant.

Ethics

Written informed consent was obtained from each patient prior to initiation of this study. This study was approved by the Ethics Committee of the First Affiliated Hospital of China Medical University.

\section{Results}

\section{Patient' background}

A total of 143 patients with LAC (100 men [69.9\%], 43 women [30.1\%]; median age 57 years, range 29-78 years) were enrolled on this study (Table 1 ). Only 60 patients $(42.0 \%)$ were nonsmokers (fewer than 100 cigarettes in their lifetime [22]). On histological examination, there were 81 patients $(56.6 \%)$ with tumor diameter $>3.0 \mathrm{~cm}$, and the remaining 62 patients (43.4\%) with tumor diameter $\leq 3.0 \mathrm{~cm}$. Tumors were poorly differentiated in 54 patients (37.8\%), moderately differentiated in 65 patients (45.5\%), and well differentiated in the remaining 24 patients (16.8\%). Additionally, 77 patients $(53.8 \%)$ had positive lymph node metastasis; the remaining 66 patients (46.2\%) had negative lymph nodes. Eighty-nine patients (62.2\%) were classified as TNM stage I-II and $54(37.8 \%)$ as TNM stage

Table 1. Correlations between MARCH9 expression and clinicopathologial characteristics in LAC patients. Note: * Statistically significant by chi-square test. Abbreviations: MARCH9, Membrane Associated Ring-CH-Type Finger 9; LAC, Lung Adenocarcinoma; LN, Lymph Node

\begin{tabular}{|c|c|c|c|c|}
\hline \multirow{2}{*}{ Variables } & \multirow{2}{*}{$\begin{array}{c}\text { Cases } \\
(\mathrm{n}=143)\end{array}$} & \multicolumn{2}{|c|}{ MARCH9 expression } & \multirow{2}{*}{$P$ value } \\
\hline & & Low $(n=75)$ & $\operatorname{High}(\mathrm{n}=68)$ & \\
\hline Gender & & & & 0.597 \\
\hline Female & 43 & 24 & 19 & \\
\hline Male & 100 & 51 & 49 & \\
\hline Age (years) & & & & 0.760 \\
\hline$\leq 60$ yrs & 65 & 35 & 30 & \\
\hline$>60$ yrs & 78 & 40 & 38 & \\
\hline Smoking & & & & 0.239 \\
\hline No & 60 & 28 & 32 & \\
\hline Yes & 83 & 47 & 36 & \\
\hline Tumor size & & & & 0.395 \\
\hline$\leq 3.0 \mathrm{~cm}$ & 62 & 30 & 32 & \\
\hline$>3.0 \mathrm{~cm}$ & 81 & 45 & 36 & \\
\hline Differentiation & & & & $<0.001 *$ \\
\hline Well/Moderate & 89 & 35 & 54 & \\
\hline Poor & 54 & 40 & 14 & \\
\hline LN metastasis & & & & $0.004^{*}$ \\
\hline Negative & 66 & 26 & 40 & \\
\hline Positive & 77 & 49 & 28 & \\
\hline TNM stage & & & & $0.021^{*}$ \\
\hline I-II & 89 & 40 & 49 & \\
\hline III-IV & 54 & 35 & 19 & \\
\hline
\end{tabular}


A

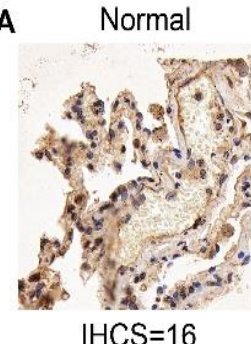

$\mathrm{IHCS}=16$
TNM stage I

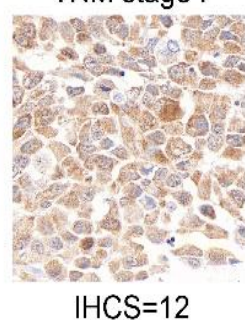

IHCS $=12$

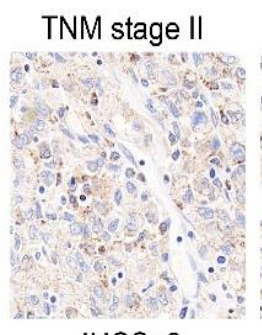

$\mathrm{IHCS}=8$
TNM stage III

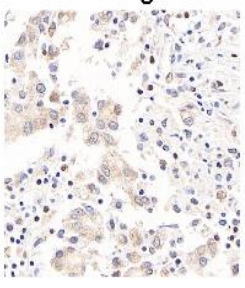

IHCS $=6$
TNM stage IV

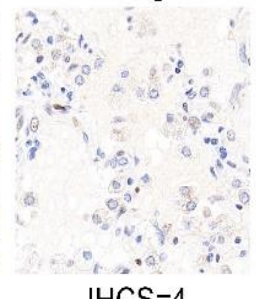

$\mathrm{IHCS}=4$
B

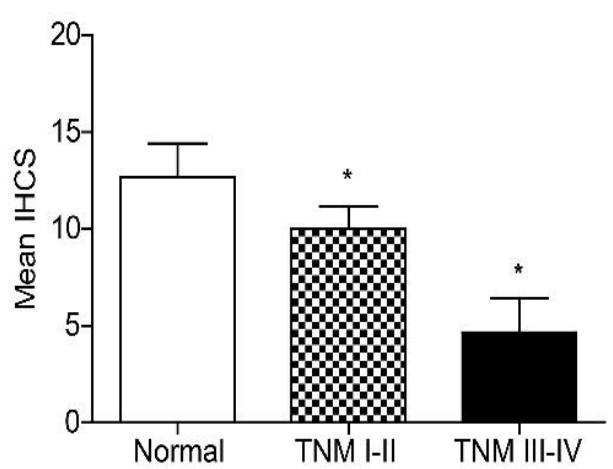

C

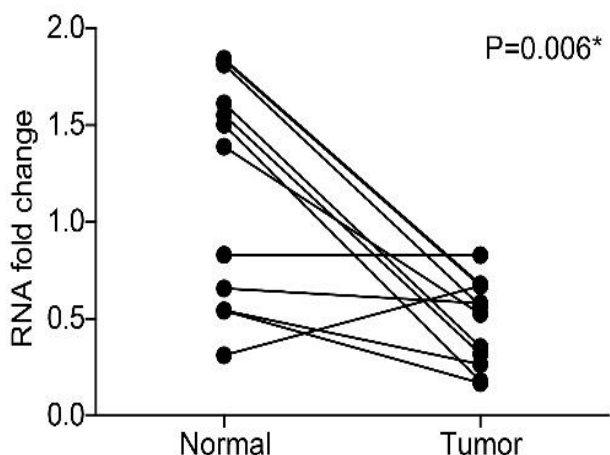

D
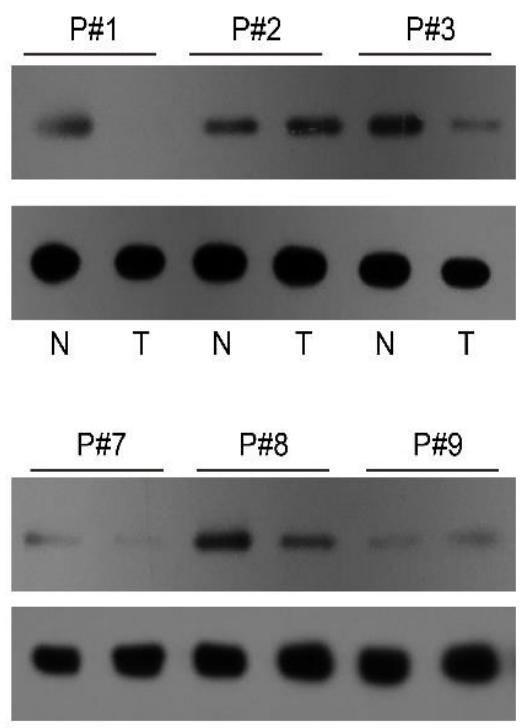

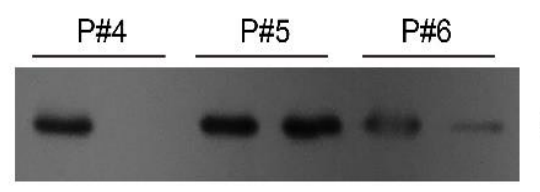

MARCH9

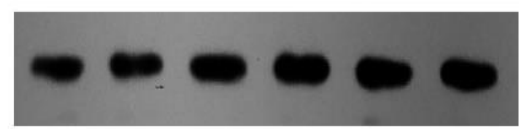

$\beta$-actin
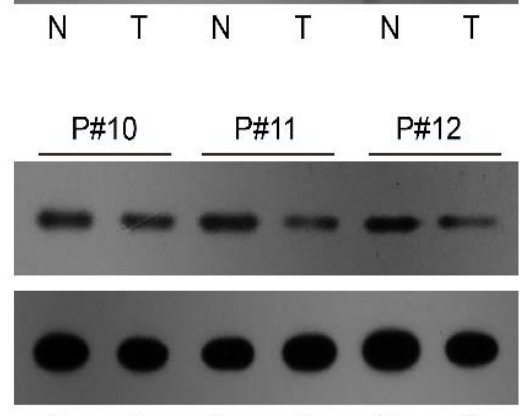

MARCH9

$\beta$-actin

$$
\begin{array}{llllll}
\mathrm{N} & \mathrm{T} & \mathrm{N} & \mathrm{T} & \mathrm{N} & \mathrm{T}
\end{array}
$$

$\begin{array}{llllll}\mathrm{N} & \mathrm{T} & \mathrm{N} & \mathrm{T} & \mathrm{N} & \mathrm{T}\end{array}$

Fig. 1. MARCH9 is downregulated in LAC tissues. (A) MARCH9 protein expression levels in paraffinized lung tissues and LAC tissues assessed using IHC, showing positive immunoreactivity in the cytoplasm. (B) Comparison of average MARCH9 IHC scores among different TNM stages, showing negative correlation of MARCH9 with tumor progression. (C) MARCH9 mRNA levels in 12 fresh-resected LAC tissues and paired adjacent lung tissues analyzed using RT-PCR normalized with GAPDH and indicating that MARCH9 is downregulated in tumor tissues. (D) Protein extracted from the 12 tissue pairs subjected to Western blot analysis with MARCH9 antibody; 9 pairs (9/12, 75.0\%) show lower MARCH9 protein levels in LAC tissues compared with lung tissues. ${ }^{*} \mathrm{P}<0.05$ compared with normal group, Student's t-test.

\section{KARGER}


III-IV. Median follow-up period was 37 months (range 13-86 months). In total, 61 patients $(42.7 \%)$ died during the study period.

\section{MARCH9 is downregulated in LAC tumor tissues}

We assessed the levels of MARCH9 protein in all 143 pairs of LAC tissues and adjacent normal liver tissues using IHC. MARCH9 protein was mainly located in the cytoplasm (Fig. 1A). Unlike the high endogenous expression in normal lung tissues, MARCH9 showed decreased levels in LAC tumor tissues and this was negatively correlated with tumor stage (Fig. 1B). We also assessed MARCH9 mRNA levels in fresh-frozen tissue pairs and found lower MARCH9 transcription in tumor tissues (Fig. 1C). Consistent with this, western blotting revealed decreased MARCH9 protein levels in tumor tissues compared with levels in adjacent normal lung tissues (Fig.
Table 2. Univariate analysis of clinicopatholgical factors for the overall survival in 143 LAC patients. Note: * Statistically significant by log-rank test. Abbreviations: LAC, Lung Adenocarcinoma; LN, Lymph Node; MARCH9, Membrane Associated Ring-CH-Type Finger 9

\begin{tabular}{|c|c|c|c|c|}
\hline \multirow{2}{*}{ Variables } & \multirow{2}{*}{$\begin{array}{c}\text { Cases } \\
(n=143)\end{array}$} & \multicolumn{2}{|c|}{ Overall survival (months) } & \multirow{2}{*}{$P$ value } \\
\hline & & Mean \pm sd & 5-year OS & \\
\hline Gender & & & & 0.072 \\
\hline Female & 43 & $60.7 \pm 3.9$ & $65.4 \%$ & \\
\hline Male & 100 & $54.1 \pm 3.1$ & $49.8 \%$ & \\
\hline Age (years) & & & & $0.030^{*}$ \\
\hline$\leq 60 \mathrm{yrs}$ & 65 & $60.7 \pm 3.2$ & $63.9 \%$ & \\
\hline$>60 \mathrm{yrs}$ & 78 & $52.7 \pm 3.4$ & $47.9 \%$ & \\
\hline Smoking & & & & 0.179 \\
\hline No & 60 & $61.7 \pm 3.9$ & $63.2 \%$ & \\
\hline Yes & 83 & $52.6 \pm 2.9$ & $49.7 \%$ & \\
\hline Tumor size & & & & $0.025^{*}$ \\
\hline$\leq 3.0 \mathrm{~cm}$ & 62 & $64.2 \pm 3.7$ & $68.5 \%$ & \\
\hline$>3.0 \mathrm{~cm}$ & 81 & $49.2 \pm 2.8$ & $44.1 \%$ & \\
\hline Differentiation & & & & $0.018^{*}$ \\
\hline Well/Moderate & 89 & $62.8 \pm 3.2$ & $62.1 \%$ & \\
\hline Poor & 54 & $48.1 \pm 3.3$ & $43.9 \%$ & \\
\hline LN metastasis & & & & $0.003^{*}$ \\
\hline Negative & 66 & $66.7 \pm 3.7$ & $75.7 \%$ & \\
\hline Positive & 77 & $48.3 \pm 2.9$ & $41.5 \%$ & \\
\hline TNM stage & & & & $<0.001^{*}$ \\
\hline I-II & 89 & $67.1 \pm 3.2$ & $70.1 \%$ & \\
\hline III-IV & 54 & $42.5 \pm 3.0$ & $33.7 \%$ & \\
\hline MARCH9 expression & & & & $0.002^{*}$ \\
\hline Positive & 75 & $48.5 \pm 2.9$ & $46.6 \%$ & \\
\hline Negative & 68 & $67.4 \pm 3.6$ & $65.3 \%$ & \\
\hline
\end{tabular}

1D). Because the MARCH9 expression pattern indicated its potential as a tumor suppressor, we then performed statistical analysis to explore its clinical significance.

Using the chi-square test, we compared patients in the low- and high-MARCH9 expression groups. We found that low MARCH9 correlated with poorly differentiated tumors $(P<0.001$; Table 1). Moreover, patients with positive lymph nodes or advanced TNM stages showed lower MARCH9 levels $(P=0.004$ and $P=0.021$, respectively). In contrast, no significant difference was found between MARCH9 expression level and sex, age, smoking history, or tumor size.

\section{Low MARCH9 expression indicates poor overall survival in LAC patients}

We plotted overall survival curves using Kaplan-Meier analysis based on different variables (Table 2, Fig. 2). Overall survival in LAC patients was not significantly correlated with sex $(P=0.072)$ or smoking history $(P=0.179)$. However, older patients showed unfavorable clinical outcomes $(P=0.030)$. As expected, shorter overall survival time was associated with larger tumor size $(P=0.025)$, poor histological differentiation $(P=0.018)$, positive lymph nodes $(P=0.003)$, and advanced TNM stages $(P<0.001)$. Importantly, a higher MARCH9 level was beneficial for overall survival in LAC patients (overall survival time $67.4 \pm 3.6$ vs $48.5 \pm 2.9$ months, $P=0.002$ ).

To enlarge the sample size and minimize bias from the a single-center sample, we further evaluated the prognostic effect of MARCH9 from a public database using a Kaplan-Meier plotter (http://www.kmplot.com). The microarray data showed that a higher MARCH9 mRNA level was correlated with better overall survival in NSCLC (Fig. 3A, $P=0.013$ ) or LAC (Fig. 3B, $P=0.037$ ). However, no prognostic significance was observed in patients with lung squamous cell carcinoma (Fig. 3C, $P=0.64$ ). Consistent with our data, the public database showed a promising role of MARCH9 in predicting clinical outcomes of LAC patients, therefore, we further focused on LAC cases in this study.

Multivariate analysis was performed to identify independent prognostic factors of LAC (Table 3). Lymph node metastasis (hazard ratio [HR] $=3.223,95 \%$ confidence interval [CI]

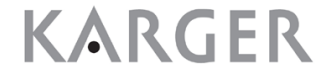



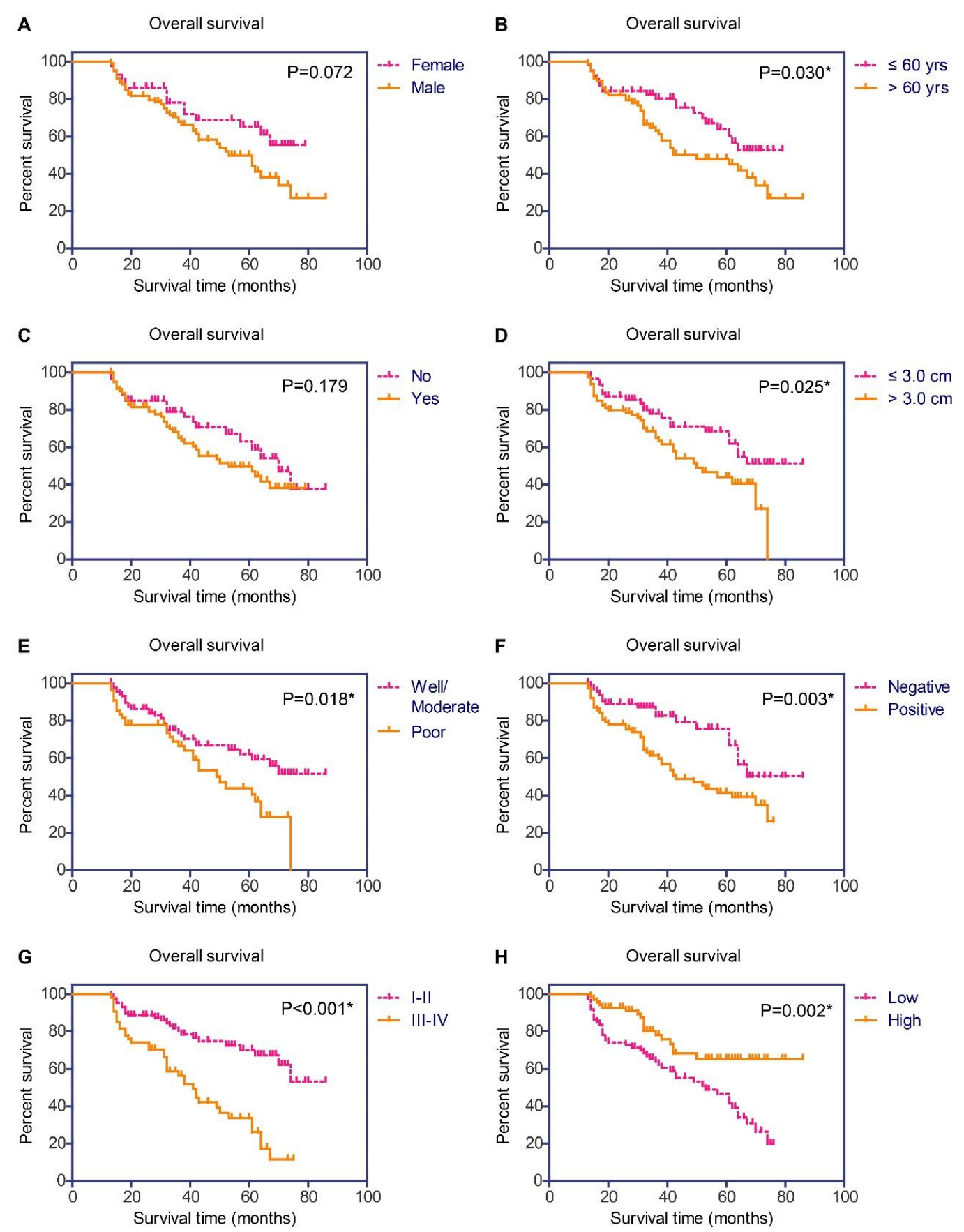

Fig. 2. MARCH9 expression level is useful in predicting clinical outcomes in LAC patients. Kaplan-Meier survival analysis based on patients' sex (A), age (B), smoking history (C), tumor size (D), tumor differentiation (E), lymph node metastasis (F), TNM stage (G), and MARCH9 expression (H). ${ }^{*} \mathrm{P}<0.05$, log-rank test. 
1.730-6.005, $P<0.001$ ), TNM stage (HR $=4.101,95 \%$ CI 2.244-7.496, $P<0.001$ ), and MARCH9 (HR $=0.342,95 \%$ CI 0.148 $0.960, P=0.048$ ) protein levels were all independent prognostic factors for overall survival in LAC patients. No independent effect was observed for age, tumor size, or tumor differentiation.

MARCH9 has no significant effect on proliferation or apoptosis of LAC cells

The clinical data revealed an independent effect of MARCH9 in predicting patients' survival, so we performed cellular experiments to explore its functional mechanisms. Endogenous MARCH9 protein expression levels were tested in HBE cells, A549 cells, and H1299 cells. HBE cells represent normal lung cells, A549 cells were derived from primary lung adenocarcinoma, and H1299 cells originated from a metastatic lung adenocarcinoma lesion. According to the western blot results, HBE cells showed the highest MARCH9 protein levels, while A549 and H1299 cells showed lower MARCH9 levels (Fig. 4A), which was consistent with the clinical findings. Therefore, we induced MARCH9 gene overexpression in both A549 and H1299 cells (Fig. 4B, 4C).

Proliferation of A549 and H1299 cells was evaluated using MTT assays (Fig. 4D, 4E; $P>0.05$ ). Overexpression of $M A R C H 9$ showed no significant effect on cell proliferation, which was further verified by colony formation experiments (Fig. 4F, 4G; $P>0.05$ ). In addition, we examined the cell cycle in MARCH9-overexpressing cells using flow cytometry; neither A549 nor H1299 showed significant alteration in cell cycle phases (Fig. 5A, 5B). Apoptosis in both cell lines was analyzed, and MARCH9 overexpression showed no apoptotic effect (Fig. 5C, 5D). The minimal effect of MARCH9 on cell proliferation or apoptosis was also consistent with the clinical data showing that MARCH9 had no significant correlation with tumor size (Table $1, P=$ 0.395 ).

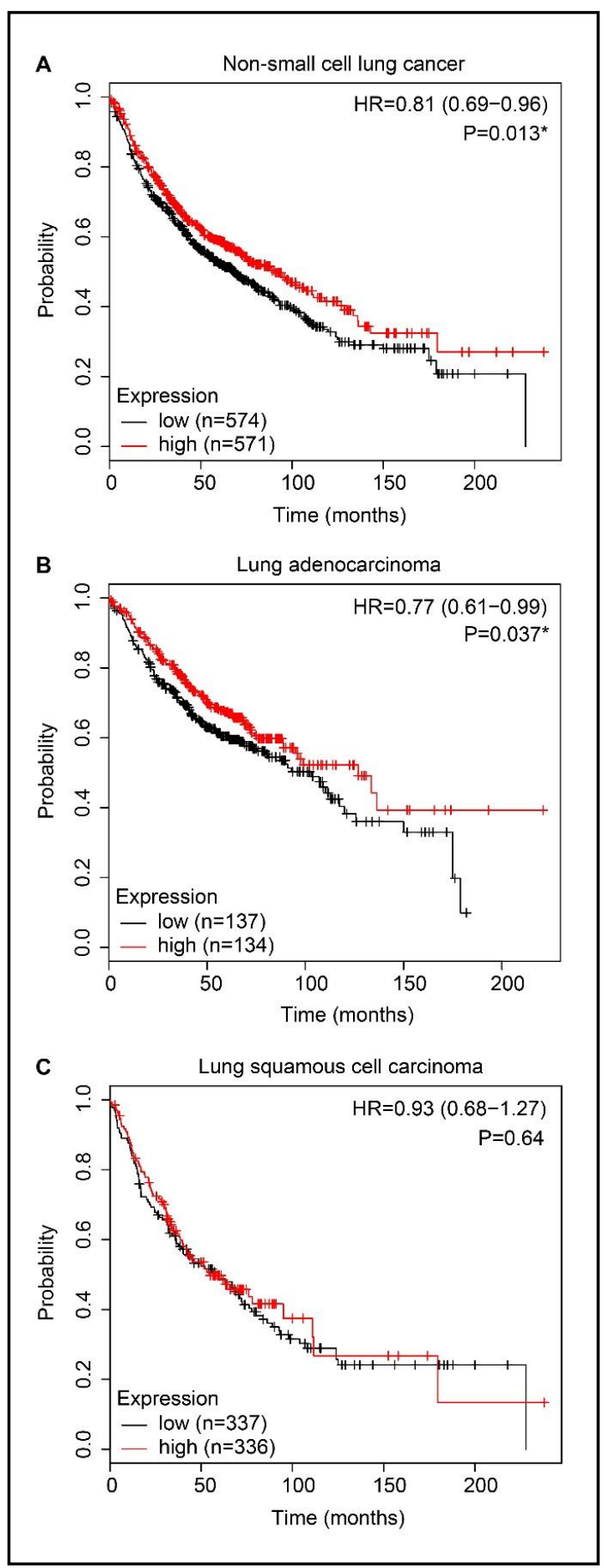

Fig. 3. Low MARCH9 is correlated with poor overall survival of LAC patients. Low MARCH9 accumulation significantly correlates with poor overall survival in NSCLC patients (A) and LAC patients (B). MARCH9 shows no clinical significance in survival in lung squamous cell carcinoma (C). Data were generated from a public database through http://www.kmplot. com. $* \mathrm{P}<0.05$, log-rank test. 
Table 3. Cox multivariate analysis of variables affecting overall survival in LAC patients. Note: * Statistically significant. Abbreviations: LAC, Lung Adenocarcinoma; LN, Lymph Node; MARCH9, Membrane Associated Ring-CH-Type Finger 9

\begin{tabular}{lccc}
\hline Variables & Hazard ratio & 95\% Confidence Interval & P value \\
\hline Age & 1.474 & $0.821-2.646$ & 0.194 \\
Tumor size & 1.508 & $0.873-2.604$ & 0.141 \\
Differentiation & 1.491 & $0.891-2.547$ & 0.139 \\
LN metastasis & 3.223 & $1.730-6.005$ & $<0.001^{*}$ \\
TNM stage & 4.101 & $2.244-7.496$ & $<0.001^{*}$ \\
MARCH9 expression & 0.342 & $0.148-0.960$ & $0.048^{*}$ \\
\hline
\end{tabular}

Fig. 4. MARCH9 overexpression has no significant effect on LAC cell proliferation. Western blot (A) analysis of MARCH9 levels in human bronchial epithelial cell line (HBE) and LAC cell lines (A549, H1299), indicating lower MARCH9 levels in tumor cells. A549 and H1299 cells transfected with MARCH9 plasmids, with transfection efficiency verified using western blot (B, C). MARCH9 overexpression shows no significant effect on cellular proliferation as revealed by MTT (D, E) and colony formation $(F, G)$ assays. Data are the mean \pm S.D. obtained from triplicate experiments. NS: no statistical significance compared with control groups transfected with plasmid vector.
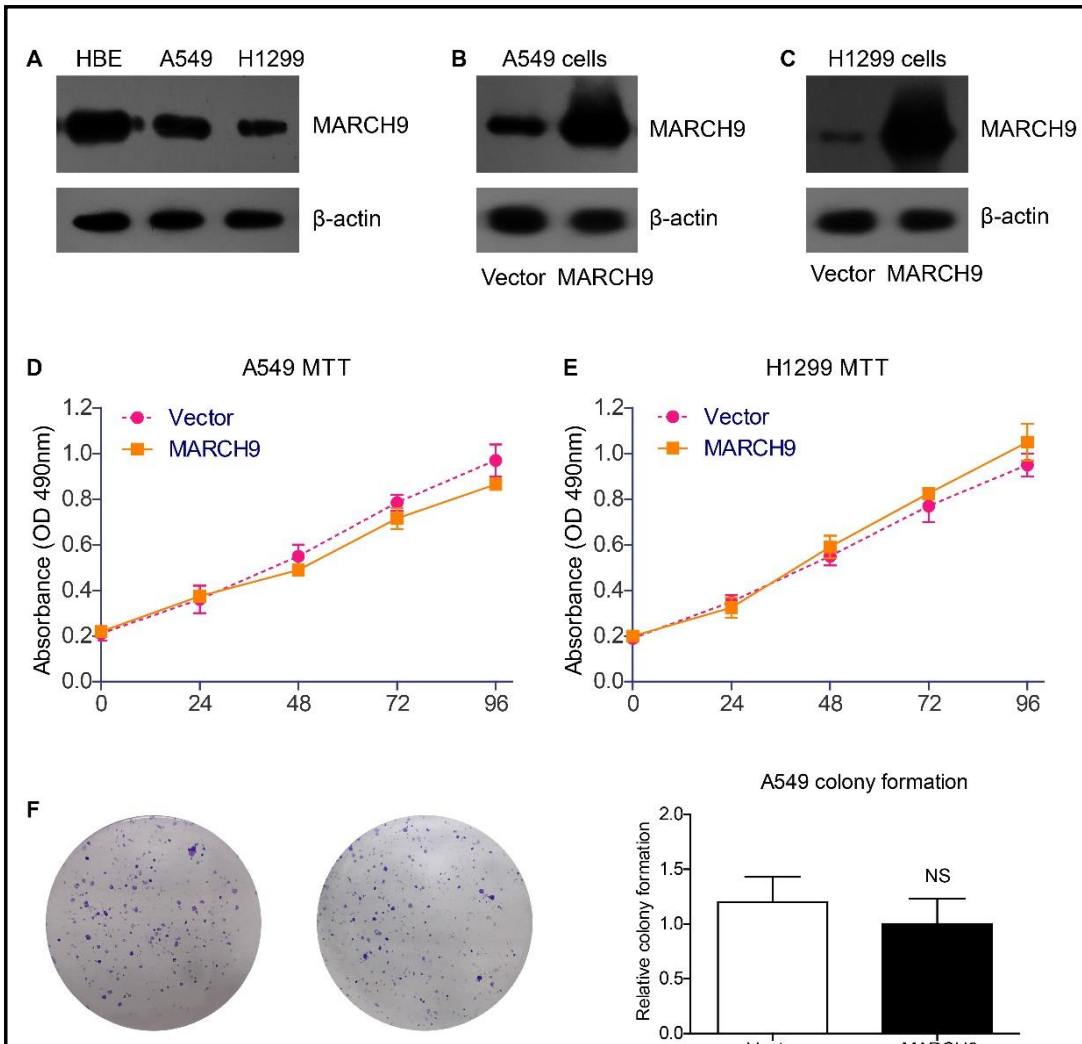

A549 colony formation

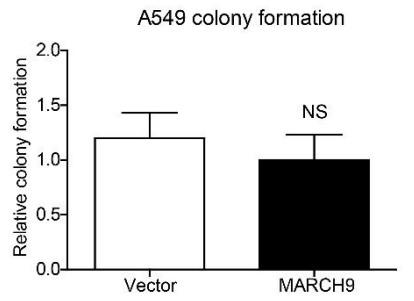

G

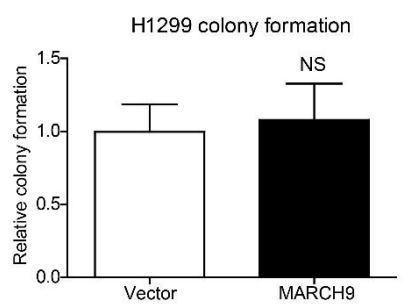




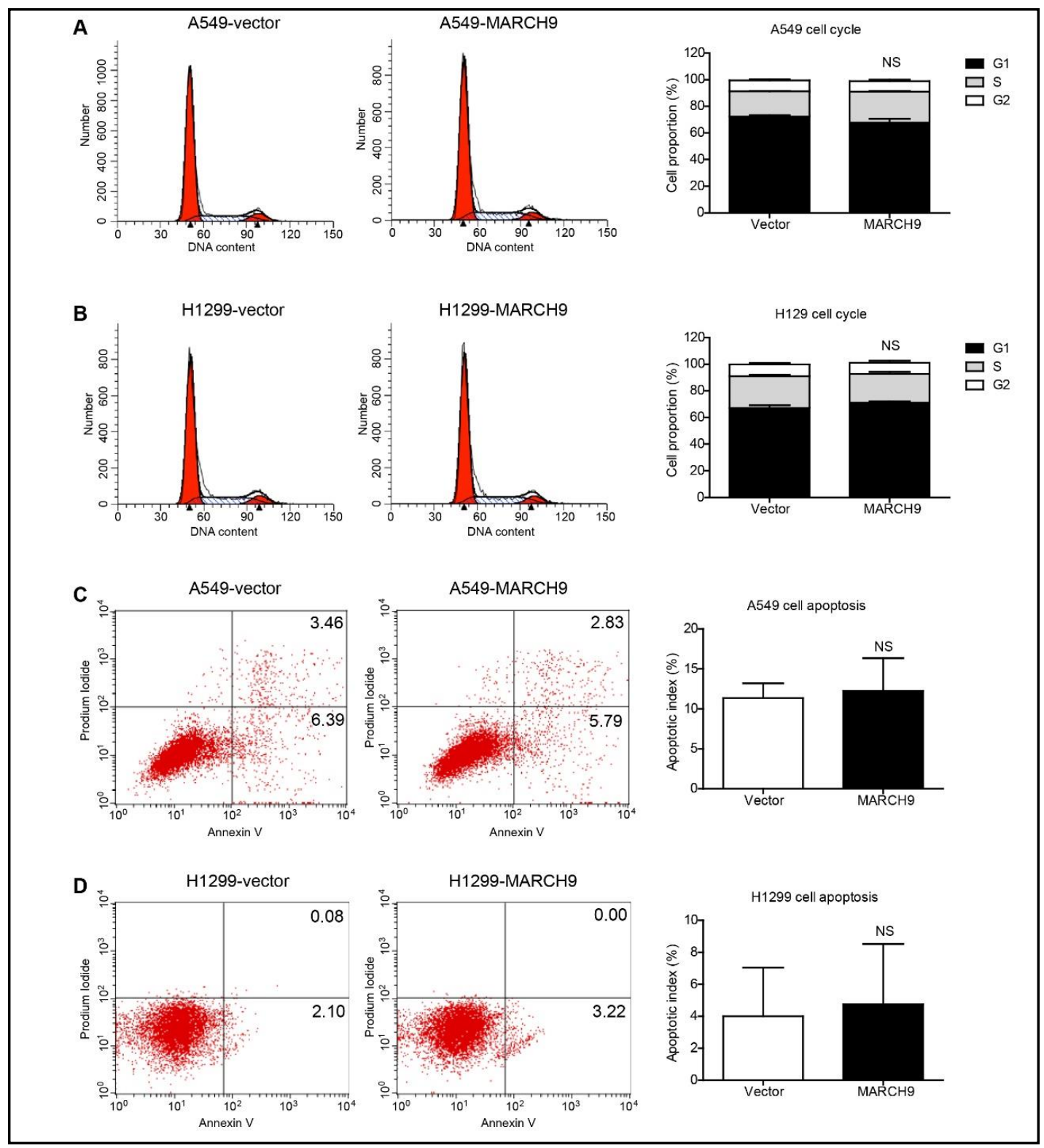

Fig. 5. MARCH9 does not affect the cell cycle or apoptosis in LAC cells. Flow cytometry demonstrating little effect of MARCH9 on cell cycle phases in A549 (A) and H1299 cells (B). Apoptosis index (C, D) tested by flow cytometry; apoptosis is not affected by MARCH9 overexpression. Data are the mean \pm S.D. obtained from triplicate experiments. NS: no statistical significance compared with control groups transfected with plasmid vector.

\section{MARCH9 overexpression represses LAC migration and invasion}

As revealed by the statistical analysis of clinical data, low MARCH9 expression was more frequently observed in patients with positive lymph nodes and advanced tumor stages (Table 1), and we therefore hypothesized that MARCH9 may be correlated with the invasion process of tumor cells. Migration of LAC cells was first monitored using wound-healing assay (Fig. 6A, 6B) and transwell assay (Fig. 6C, 6D). As expected, the migration capacity of both A549 and H1299 cells was attenuated by MARCH9 overexpression, further demonstrating its tumor-suppressing role in LAC. In addition, Matrigel-coated transwell chambers were used to assess the invasion process of LAC cells, demonstrating that MARCH9 can inhibit tumor invasion of both A549 (Fig. 6E) and H1299 (Fig. 6F) cells. 


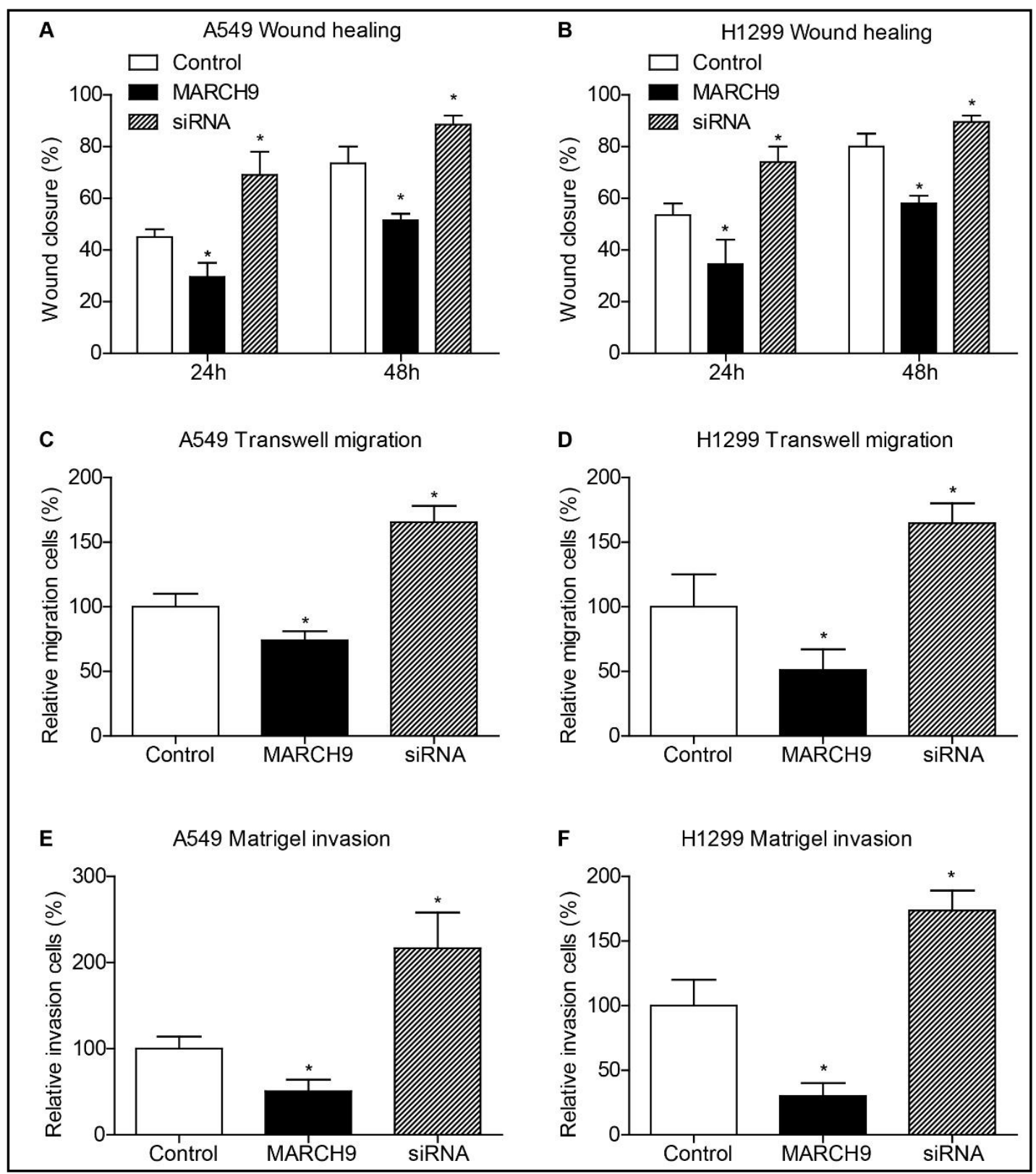

Fig. 6. MARCH9 inhibits migration and invasion of LAC cells. Wound healing experiments showing attenuation of cell migration by MARCH9 overexpression in both A549 (A) and H1299 cells (B). Migration capacity of LAC cells also verified by transwell chamber assays (C, D), showing results consistent with wound-healing assays. Matrigel-Transwell assay (E, F) confirms the role of MARCH9 overexpression in inhibiting invasion in LAC cells. * $\mathrm{P}<0.05$, Student's t-test.

MARCH9 exerts tumor suppressing functions by downregulating ICAM-1

ICAM-1, also known as CD54, is a protein expressed in the cell membrane and it plays critical roles in cell migration [23]. A previous mass spectrometry study identified ICAM-1 as a potential candidate substrate of MARCH9 [24] in lymphoid B cells, and this was also verified in 293T cells [25]. Taking into consideration that ICAM-1 plays tumor-promoting roles in lung cancer [26], we investigated potential crosstalk between MARCH9 and ICAM1 in LAC cells. IHC staining of clinical tissues identified a significant negative correlation between the expression levels of MARCH9 and ICAM-1 (Fig. 7A), indicating their intrinsic 
Fig. 7. ICAM-1 is downregulated by MARCH9. (A) ICAM-1 protein expression on IHC showing cytoplasmic and membrane localization. ICAM-1 and MARCH9 expression levels are negatively correlated. (B) LAC cells transfected with different plasmids showing that MARCH9 can downregulate ICAM-1protein levels. ICAM1 transfection increases RhoA activation; MARCH9 overexpression attenuates it. Co-immunoprecipitation results showing that MARCH9 interacts with ICAM-1 protein, consistent with IHC data. (C) MARCH9 overexpression enhances K48linked ICAM-1polyubiquitination. Experimental procedure is similar to that in panel B. (D) MG-132, a proteasome inhibitor, attenuates ICAM-1 degradation in MARCH9 overexpressing cells. Functional analysis shows that ICAM-1 overexpression increases cell migration (E) and invasion (F), whereas MARCH9 overexpression abolishes these oncogenic effects.

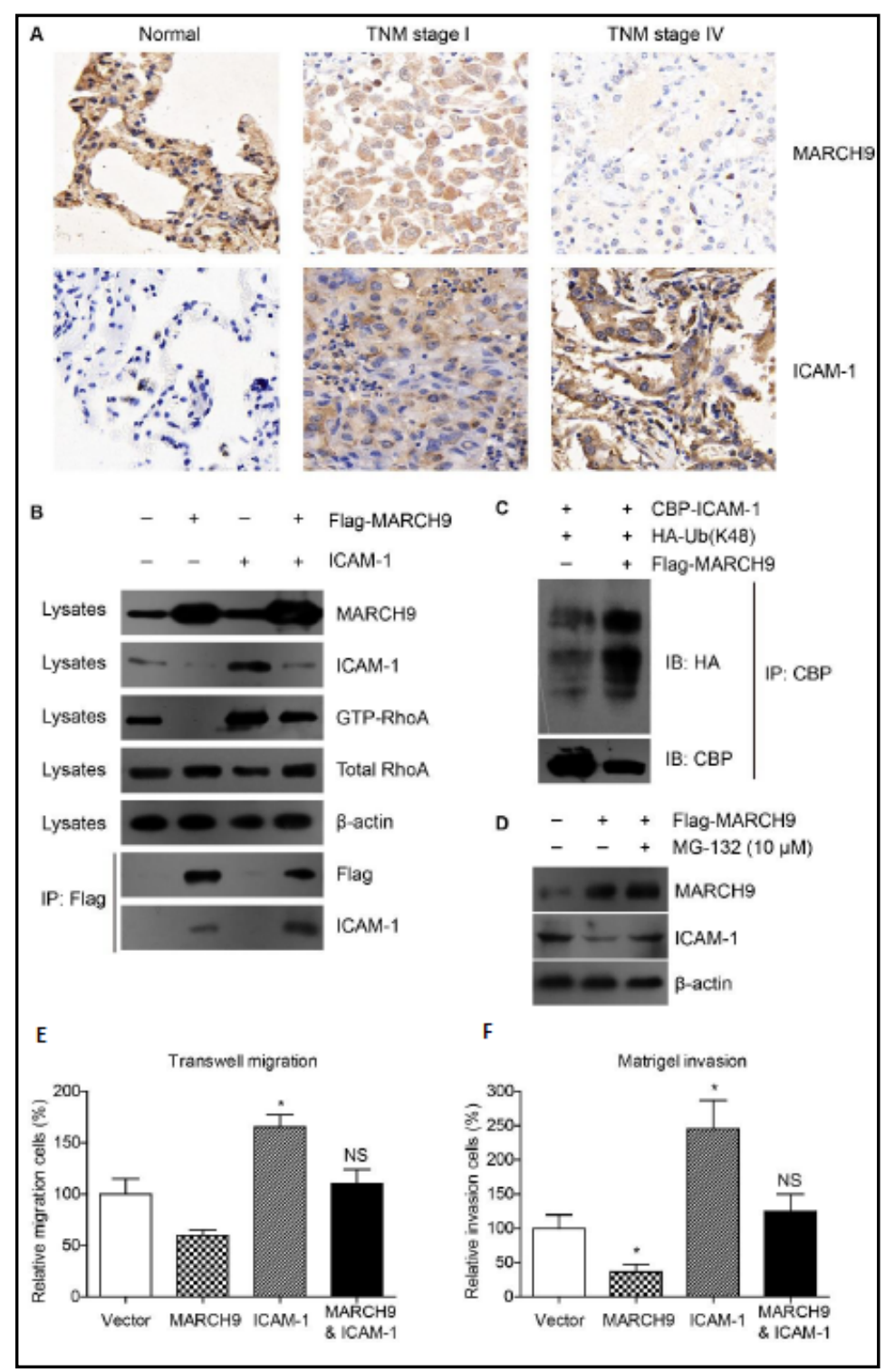

relationship. We then transfected LAC cells with both Flag-tagged MARCH9 and/or ICAM-1 to validate their direct interaction. As shown by western blot (Fig. 7B), MARCH9 overexpression significantly downregulated the ICAM-1 protein level as well as its downstream RhoA activity. Of note, immunoprecipitation experiments showed that ICAM-1 can interact with MARCH9 protein in LAC cells (Fig. 7B), confirming their enzyme-substrate crosstalk. We also performed K48-linked polyubiquitination analysis and, as expected, MARCH9 enhanced the K48-linked polyubiquitination of ICAM-1 (Fig. 7C). Meanwhile, MG-132, a proteasome inhibitor, significantly attenuated ICAM-1 degradation in MARCH9 overexpressing cells (Fig. 7D). Therefore, we conclude that ICAM-1 can be ubiquitinated and degraded downstream of MARCH9.

We also tested the antergic effects of MARCH9 and ICAM-1 in modulating LAC progression. Both migration and invasion capacities were enhanced by ICAM-1 overexpression (Fig. 7E, 7F), but co-expression of MARCH9 attenuated these tumor-promoting effects. These data indicated that MARCH9 can inhibit LAC progression at least partially by downregulating ICAM-1 oncoprotein. 


\section{Cellular Physiology Cell Physiol Biochem 2018;50:92-107

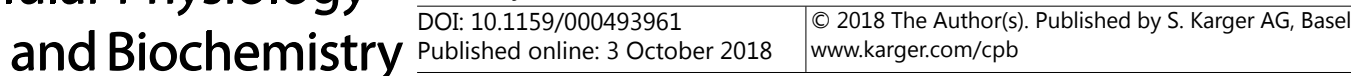 Shen et al.: MARCH9 Inhibits LAC Progression}

\section{Discussion}

Despite great achievements in diagnosis and treatment over the past few decades, lung cancer remains the leading cause of death [27]. Currently, the mostly considered factors in clinical trials are tumor differentiation, lymph node metastasis, and TNM stage. However, increasingly more studies have revealed that patients show completely distinct clinical outcomes even with the same tumor stage. Therefore, a better illustration of the molecular basis of lung cancer would be invaluable for distinguishing patients at high risk. Molecular studies will also help predict patients' prognosis and direct novel drug development.

Post-translational modification (PTM) of proteins has been recognized to play important roles in tumor development in recent decades [28, 29], and it is now attracting more attention in molecular studies. Among numerous PTMs, ubiquitination is a highly important mechanism in modulating protein activity and degradation; the process is reversible and is involved in cell differentiation and tissue development [30,31]. Ubiquitination balance is regulated by E3 ubiquitin ligases and deubiquitinases, and dysregulation of either one would lead to pathophysiologies including neoplasm. Increasing evidence has verified the prognostic and therapeutic roles of various E3 ligases in malignancy [32]. MARCH is a E3 ligase subfamily that functions by adding ubiquitin residues onto proteins, subsequently driving proteolytic degradation as other E3 ligases. All11 members of the MARCH family have long since been identified [33], and several of them show some structural homology; for example, MARCH4 and MARCH9 seem to be closely related (62\% identical at the amino acid level) and may share similar substrates [34]. While several critical substrates downstream of MARCH4 have been identified [35-37], research on MARCH9 is progressing slowly. Previous studies have shown that MARCH9 is located on the Golgi apparatus and lysosomes [36] and is predominantly expressed in lymphoid cells [38].

In this study, we have provided initial evidence of the tumor-suppressing role of MARCH9 in lung adenocarcinoma (LAC). According to our data, MARCH9 was endogenously detectable in normal lung tissues at both mRNA and protein levels. However, its expression was significantly decreased in LAC tissues and was negatively correlated with tumor metastasis and tumor stage. Both univariate and multivariate analysis revealed an independent effect of MARCH9 in predicting overall survival, which was consistent with the survival data from a public microarray database. We performed cellular experiments to investigate the functional mechanisms of MARCH9 in LAC progression. Interestingly, MARCH9 had no significant effect on either the cell cycle or cell apoptosis as revealed by MTT, colony formation, and flow cytometry assays. However, MARCH9 overexpression significantly inhibited cell migration and invasion capacity in LAC cells and thus can explain our clinical findings.

As a ubiquitin ligase, a recent study identified 13 potential MARCH9 targets in lymphoid B cells by using high throughput quantitative mass spectrometry strategies [24], and only 5 of them were further validated as novel targets of MARCH9 using flow cytometry analyses. Among them, ICAM-1 was also validated by another group, indicating that MARCH9 can directly ubiquitinate ICAM-1 and promote its degradation [25]. Since ICAM1 is a well-known oncoprotein in lung cancer, we further tested whether MARCH9 can interact and downregulate ICAM-1 in lung cancer cells. IHC examination in clinical LAC tissues showed a negative correlation between the levels of MARCH9 and ICAM-1 proteins. Immunoprecipitation experiments in LAC cells identified their direct interaction. Moreover, ICAM-1 overexpression enhanced cell aggressiveness via activating RhoA signaling, while MARCH9 co-expression abolished this tumor-promoting effect. Therefore, we have provided original evidence for MARCH9-ICAM-1 crosstalk in lung cancer progression. 


\section{Cellular Physiology Cell Physiol Biochem 2018;50:92-107

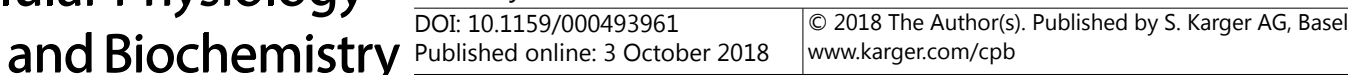 Shen et al.: MARCH9 Inhibits LAC Progression}

\section{Conclusion}

Taken together, our findings indicate that MARCH9 downregulation is correlated with poor clinical outcomes in LAC patients. MARCH9 may suppress tumor invasion by downregulating ICAM-1-RhoA signaling.

\section{Abbreviations}

CI (confidence interval); HR (hazard ratio); ICAM-1 (intercellular adhesion molecule 1); IHC (immunohistochemistry); LAC (lung adenocarcinoma); LN (lymph node); MARCH9 (membrane-associated RING-CH protein 9); OS (overall survival); PCR (polymerase chain reaction); PTM (post-translational modification); RNA (ribonucleic acid); RT-qPCR (quantitative real-time polymerase chain reaction); RhoA (Ras homolog gene family member A); TNM (tumor, nodes, metastasis).

\section{Disclosure Statement}

No conflicts of interest exists.

\section{References}

1 Zhao J, Li L, Wang Q, Han H, Zhan Q Xu M: CircRNA Expression Profile in Early-Stage Lung Adenocarcinoma Patients. Cell Physiol Biochem 2017;44:2138-2146.

-2 Zhang Q, Liu H, Zhu Q, Zhan P, Zhu S, Zhang J, Lv T, Song Y: Patterns and functional implications of platelets upon tumor "education". Int J Biochem Cell Biol 2017;90:68-80.

- Lang Y, Kong X, He C, Wang F, Liu B, Zhang S, Ning J, Zhu K, Xu S: Musashi1 Promotes Non-Small Cell Lung Carcinoma Malignancy and Chemoresistance via Activating the Akt Signaling Pathway. Cell Physiol Biochem 2017;44:455-466.

4 Yang R, Li P, Zhang G, Lu C, Wang H, Zhao G: Long Non-Coding RNA XLOC_008466 Functions as an Oncogene in Human Non-Small Cell Lung Cancer by Targeting miR-874. Cell Physiol Biochem 2017;42:126-136.

5 Garcia-Lora A, Algarra I, Garrido F: MHC class I antigens, immune surveillance, and tumor immune escape. J Cell Physiol 2003;195:346-355.

-6 De Gassart A, Camosseto V, Thibodeau J, Ceppi M, Catalan N, Pierre P, Gatti E: MHC class II stabilization at the surface of human dendritic cells is the result of maturation-dependent MARCH I down-regulation. Proc Natl Acad Sci U S A 2008;105:3491-3496.

7 Ohmura-Hoshino M, Matsuki Y, Mito-Yoshida M, Goto E, Aoki-Kawasumi M, Nakayama M, Ohara 0, Ishido S: Cutting edge: requirement of MARCH-I-mediated MHC II ubiquitination for the maintenance of conventional dendritic cells. J Immunol 2009;183:6893-6897.

$>8$ Nakamura N: The role of the transmembrane RING finger proteins in cellular and organelle function. Membranes 2011;1:354-393.

-9 Nagarajan A, Petersen MC, Nasiri AR, Butrico G, Fung A, Ruan HB: MARCH1 regulates insulin sensitivity by controlling cell surface insulin receptor levels. Nat Commun 2016;7:12639.

10 Han SO, Xiao K, Kim J, Wu JH, Wisler JW, Nakamura N, Freedman NJ, Shenoy SK: MARCH2 promotes endocytosis and lysosomal sorting of carvedilol-bound beta(2)-adrenergic receptors. J Cell Biol 2012;199:817-830.

11 Xu S, Cherok E, Das S, Li S, Roelofs BA, Ge SX, Polster BM, Boyman L, Lederer WJ, Wang C, Karbowski M: Mitochondrial E3 ubiquitin ligase MARCH5 controls mitochondrial fission and cell sensitivity to stressinduced apoptosis through regulation of MiD49 protein. Mol Biol Cell 2016;27:349-359.

12 Meng Y, Hu J, Chen Y, Yu T, Hu L: Silencing MARCH1 suppresses proliferation, migration and invasion of ovarian cancer SKOV3 cells via downregulation of NF-kappaB and Wnt/beta-catenin pathways. Oncol Rep 2016;36:2463-2470. 


\section{Cellular Physiology Cell Physiol Biochem 2018;50:92-107 and Biochemistry \begin{tabular}{l|l} 
DOI: 10.1159/000493961 & $\begin{array}{l}\text { (c) } 2018 \text { The Author(s). Published by S. Karger AG, Basel } \\
\text { www.karger.com/cpb }\end{array}$
\end{tabular} \\ Shen et al.: MARCH9 Inhibits LAC Progression}

13 Xia D, Ji W, Xu C, Lin X, Wang X, Xia Y, Lv P, Song Q, Ma D, Chen Y: Knockout of MARCH2 inhibits the growth of HCT116 colon cancer cells by inducing endoplasmic reticulum stress. Cell Death Dis 2017;8:e2957.

14 Hu J, Meng Y, Zhang Z, Yan Q Jiang X, Lv Z, Hu L: MARCH5 RNA promotes autophagy, migration, and invasion of ovarian cancer cells. Autophagy 2017;13:333-344.

15 Zhang L, Wang H, Tian L, Li H: Expression of USP7 and MARCH7 Is Correlated with Poor Prognosis in Epithelial Ovarian Cancer. Tohoku J Exp Med 2016;239:165-175.

-16 Singh S, Saraya A, Das P, Sharma R: Increased expression of MARCH8, an E3 ubiquitin ligase, is associated with growth of esophageal tumor. Cancer Cell Int 2017;17:116.

-17 Liu H, Xu Y, Zhang Q, Li K, Wang D, Li S, Ning S, Yang H, Shi W, Liu Z, Chen Y: Correlations between TBL1XR1 and recurrence of colorectal cancer. Sci Rep 2017;7:44275.

18 Liu H, Zhang Q, Li K, Gong Z, Liu Z, Xu Y, Swaney MH, Xiao K, Chen Y: Prognostic significance of USP33 in advanced colorectal cancer patients: new insights into $\beta$-arrestin-dependent ERK signaling. Oncotarget 2016;7:81223-81240.

19 Zhang Q, Yuan L, Liu D, Wang J, Wang S, Zhang Q, Gong Y, Liu H, Hao A, Wang Z: Hydrogen sulfide attenuates hypoxia-induced neurotoxicity through inhibiting microglial activation. Pharmacol Res 2014;84:32-44.

20 Liu H, Liu Z, Li K, Li S, Song L, Gong Z, Shi W, Yang H, Xu Y, Ning S: TBL1XR1 predicts isolated tumor cells and micrometastasis in patients with TNM stage I/II colorectal cancer. J Gastroenterol Hepatol 2017;32:15701580.

-21 Xu Y, Yang X, Li Z, Li S, Guo S, Ismail S, Liu H, Huang Z, Zhang Z, Chen Y, Sun Q: Sprouty2 correlates with favorable prognosis of gastric adenocarcinoma via suppressing FGFR2-induced ERK phosphorylation and cancer progression. Oncotarget 2017;8:4888-4900.

-22 Subramanian J, Govindan R: Lung cancer in never smokers: a review. J Clin Oncol 2007;25:561-570.

-23 Yang L, Froio RM, Sciuto TE, Dvorak AM, Alon R, Luscinskas FW: ICAM-1 regulates neutrophil adhesion and transcellular migration of TNF- $\alpha$-activated vascular endothelium under flow. Blood 2005;106:584-592.

24 Hor S, Ziv T, Admon A, Lehner PJ: Stable isotope labeling by amino acids in cell culture and differential plasma membrane proteome quantitation identify new substrates for the MARCH9 transmembrane E3 ligase. Mol Cell Proteomics 2009;8:1959-1971.

25 Hoer S, Smith L, Lehner PJ: MARCH-IX mediates ubiquitination and downregulation of ICAM-1. FEBS Lett 2007;581:45-51.

26 Kotteas EA, Boulas P, Gkiozos I, Tsagkouli S, Tsoukalas G, Syrigos KN: The intercellular cell adhesion molecule-1 (icam-1) in lung cancer: implications for disease progression and prognosis. Anticancer Res 2014;34:4665-4672.

27 Siegel RL, Miller KD, Jemal A: Cancer statistics, 2016. CA Cancer J Clin 2016;66:7-30.

28 Bode AM, Dong Z: Post-translational modification of p53 in tumorigenesis. Nat Rev Cancer 2004;4:793.

-29 Dai C, Gu W: p53 post-translational modification: deregulated in tumorigenesis. Trends Mol Med 2010;16:528-536.

-30 Fraile J, Quesada V, Rodríguez D, Freije J, López-otín C: Deubiquitinases in cancer: new functions and therapeutic options. Oncogene 2012;31:2373.

-31 D’Arcy P, Linder S: Proteasome deubiquitinases as novel targets for cancer therapy. Int J Biochem Cell Biol 2012;44:1729-1738.

-32 Nakayama KI, Nakayama K: Ubiquitin ligases: cell-cycle control and cancer. Nat Rev Cancer 2006;6:369.

-33 Samji T, Hong S, Means RE: The membrane associated RING-CH proteins: a family of E3 ligases with diverse roles through the cell. Int Sch Res Notices 2014;2014:637295.

34 Ohmura-Hoshino M, Goto E, Matsuki Y, Aoki M, Mito M, Uematsu M, Hotta H, Ishido S: A novel family of membrane-bound E3 ubiquitin ligases. J Biochem 2006;140:147-154.

35 Bartee E, Mansouri M, Nerenberg BTH, Gouveia K, Früh K: Downregulation of major histocompatibility complex class I by human ubiquitin ligases related to viral immune evasion proteins. J Virol 2004;78:11091120 .

-36 Bartee E, Eyster CA, Viswanathan K, Mansouri M, Donaldson JG, Früh K: Membrane-Associated RING-CH proteins associate with Bap31 and target CD81 and CD44 to lysosomes. PloS One 2010;5:e15132.

-37 Nice TJ, Deng W, Coscoy L, Raulet DH: Stress-regulated targeting of the NKG2D ligand Mult1 by a membrane-associated RING-CH family E3 ligase. J Immunol 2010;185:5369-5376.

-38 Su AI, Cooke MP, Ching KA, Hakak Y, Walker JR, Wiltshire T, Orth AP, Vega RG, Sapinoso LM, Moqrich A: Large-scale analysis of the human and mouse transcriptomes. Proc Natl Acad Sci U S A 2002;99:4465-4470. 